\title{
Actividad antibacteriana de diatomeas marinas aisladas de Acapulco, Guerrero, México
}

\author{
Antibacterial activity of marine diatoms isolated from Acapulco, Guerrero, Mexico
}

\author{
Cynthia Nájera-Arce ${ }^{1}$, Patricia Álvarez-Fitz², Donaciano Pérez-Castro³, Jeiry Toribio- \\ Jiménez $^{1}$ y Natividad Castro-Alarcón ${ }^{1 *}$
}

\begin{abstract}
${ }^{1}$ Laboratorio de Investigación en Microbiología, Facultad de Ciencias Químico Biológicas, Universidad Autónoma de Guerrero, Lázaro Cárdenas S/N Col. La Haciendita, Chilpancingo, Guerrero, México. *Autor de correspondencia: natividadcastro24@gmail.com

${ }^{2}$ Cátedras CONACyT - UAGro, Laboratorio de Toxicología y Salud Ambiental, Facultad de Ciencias Químico Biológicas, Universidad Autónoma de Guerrero, Av. Lázaro Cárdenas s/n Col. La Haciendita, Chilpancingo, Guerrero, México ${ }^{3}$ Laboratorio de Biotecnología e Impacto Ambiental, Escuela Superior de Ciencias Ambientales, Universidad Autónoma de Guerrero, Carretera Cayaco-Puerto Marques (Ejido Llano Largo 56, 57 y 58), Acapulco, Guerrero, México
\end{abstract}

\begin{abstract}
Marine diatoms synthesize and secrete a variety of secondary metabolites with potential applications in the pharmaceutical industry, however their study is still limited. Therefore, the objective of this work was to isolate and culture the species Chaetoceros curvisetus, Asterionella japonica, and Biddulphia mobiliensis from Santa Lucía Bay in Acapulco, Guerrero, Mexico, as well as to determine the antibacterial activity against bacteria of clinical importance and the analysis of qualitative phytochemical profile of their extracts obtained. Biomass yield was $219.62 \pm 0.99 ; 151.12 \pm$ 1.41 , and $109.04 \pm 1.48 \mathrm{mg}$ for C. curvisetus, A. japonica, and B. mobiliensis, respectively. Hexane, dichloromethane and acetone extracts were obtained for each species and the qualitative phytochemical profile indicated that these marine diatoms were rich in essential oils, alkaloids, and terpenes. C. curvisetus extract was observed to possess the highest antibacterial activity with Minimum Inhibitory Concentrations (MIC) between 0.5 and $2 \mathrm{mg} \mathrm{mL}^{-1}$ for ATCC bacteria, and 8 and $16 \mathrm{mg} \mathrm{mL}^{-1}$ for E. coli BLEE-producing strains.
\end{abstract}

Key words: Chaetoceros curvisetus, Asterionella japonica, Biddulphia mobiliensis, secondary metabolites, phytochemical profile

Resumen.- Las diatomeas marinas sintetizan y secretan una gran variedad de metabolitos secundarios con aplicaciones potenciales en la industria farmacéutica, sin embargo su estudio aún es limitado. Por tal motivo, el objetivo de este trabajo fue aislar y cultivar las especies Chaetoceros curvisetus, Asterionella japonica, y Biddulphia mobiliensis de la Bahía Santa Lucía del Puerto de Acapulco, Guerrero, México, así como determinar la actividad antibacteriana frente a bacterias de importancia clínica y analizar el perfil fitoquímico cualitativo de sus extractos totales. La biomasa fue de $219,62 \pm 0,99$; $151,12 \pm 1,41$ y 109,04 $\pm 1,48 \mathrm{mg}$ para C. curvisetus, A. japonica, y B. mobiliensis, respectivamente. Extractos con hexano, diclorometano, y acetona fueron obtenidos de cada especie, y el perfil fitoquímico cualitativo indicó que estas diatomeas marinas fueron ricas en aceites esenciales, alcaloides, y terpenos. Se observó que los extractos de $C$. curvisetus poseen alta actividad con Concentraciones Mínimas Inhibitorias (CMI) entre 0,5 y $2 \mathrm{mg} \mathrm{mL}^{-1}$ para bacterias tipo ATCC y de 8 y $16 \mathrm{mg}$ $\mathrm{mL}^{-1}$ para cepas de $E$. coli productoras de BLEE.

Palabras clave: Chaetoceros curvisetus, Asterionella japonica, Biddulphia mobiliensis, metabolitos secundarios, perfil fitoquímico

\section{INTRODUCCIÓN}

Las diatomeas marinas son consideradas como fuentes renovables que sintetizan, acumulan y secretan una gran variedad de metabolitos estructuralmente nuevos y biológicamente activos como polisacáridos, policétidos, terpenoides, esteroles, ácidos grasos, compuestos fenólicos y pigmentos. Estos compuestos han despertado el interés de muchos investigadores debido a su actividad antimicrobiana, anticancerígena y antiinflamatoria (GómezLuna 2007, Jyotirmayee et al. 2014).

Se estima que en México existe un número de aproximadamente 5,000 especies de diatomeas marinas en aguas del Pacífico y Golfo de México (HernándezBecerril 2014). En la Bahía Santa Lucía del Puerto de Acapulco, Guerrero se han aislado e identificado 88 géneros y 458 especies (Meave del Castillo et al. 2012), 
de las cuales sólo se tienen algunos estudios sobre la biodiversidad, distribución, taxonomía y variación temporal. Dentro de las especies aisladas con mayor frecuencia se encuentran Chaetoceros curvisetus Cleve, 1889 y Asterionella japonica Cleve \& Möller, 1882 (Meave del Castillo et al. 2012, Rojas-Herrera et al. 2012, MorenoDíaz et al. 2015), pero también se han identificado especies de Biddulphia como B. alternans, B. biddulphiana y $B$. tridens (Meave del Castillo et al. 2012). No se tienen reportes previos en la literatura sobre el aislamiento, distribución y variación temporal en esta bahía de Biddulphia mobiliensis Van Heurck, 1882.

C. curvisetus, es una diatomea céntrica caracterizada por poseer válvulas bipolares y estructuras denominadas setas las cuales tienen paredes celulares de sílice muy gruesas y se fusionan para formar cadenas largas y circulares que miden de 8 a $20 \mathrm{~mm}$ de longitud (Lee \& Lee 2011). Ha sido aislada desde septiembre a enero con temperaturas entre 24 y $30{ }^{\circ} \mathrm{C}$ (Rojas-Herrera et al. 2012, Moreno-Díaz et al. 2015). Esta diatomea se ha utilizado ampliamente en la acuicultura como suplemento alimenticio de crustáceos y peces de interés comercial (Sunesen et al. 2008). Wang et al. (2010), demostraron que $C$. curvisetus contiene una fracción lipídica y algunos ácidos grasos que le confieren actividad antimicrobiana contra Bacillus subtilis, Staphylococcus aureus, Escherichia coli, Proteus vulgaris, Saccharomyces cerevisiae y Candida albicans.

A. japonica es una diatomea pennada cuyas células heteropolares están unidas en colonias con forma de estrella de $160 \mathrm{~mm}$ de diámetro, representa el 90\% de la población del fitoplancton marino (Round et al. 2007), y de acuerdo a Rojas-Herrera et al. (2012), se puede encontrar en diciembre a una temperatura de aproximadamente $25^{\circ} \mathrm{C}$. Se ha utilizado como un excelente indicador de la calidad del agua (Pal \& Choudhury 2014). Viso et al. (1987) evidenciaron la actividad antimicrobiana de esta especie contra Staphylococcus aureus, Micrococcus luteos, Sarcina sp., Candida pseudotropicalis, Trichophyton rubrum y Fusarium fulvum. Además, se han aislado algunos compuestos como carotenoides, glucolípidos y ácidos grasos a partir de ella (Yongmanitchai \& Ward 1989).

B. mobiliensis es una diatomea céntrica formada por dos valvas con espinas que salen de los márgenes apicales y del centro de las mismas, mide 100 a $250 \mu \mathrm{m}$ y posee numerosos cloroplastos periféricos así como un núcleo central (Round et al. 2007). Volkman et al. (1980), reportaron la presencia de esteroles y ácidos grasos en esta especie y de acuerdo a lo reportado por Walter \&
Mahesh (2000), presenta actividad antibacteriana frente a Bacillus subtilis, Staphylococcus aureus, Escherichia coli, Klebsiella pneumoniae, Pseudomonas aeruginosa, Salmonella sp. y Candida sp.

La Bahía Santa Lucía es uno de los sitios turísticos más importantes de México, sin embargo se sabe poco sobre su riqueza fitoplanctónica y características biológicas, por lo que es importante realizar estudios que permitan aprovechar mejor este recurso natural. Con base en lo anterior, la hipótesis de este trabajo es que los extractos crudos de las diatomeas marinas C. curvisetus, A. japonica y B. mobiliensis poseen compuestos capaces de inhibir el crecimiento bacteriano, y por tal motivo el objetivo de este estudio fue aislar y cultivar estas especies a partir de clones de muestras planctónicas de la Bahía Santa Lucía del Puerto de Acapulco, Gro. México, con la finalidad de determinar el perfil fitoquímico cualitativo y evaluar la actividad antibacteriana de extractos totales obtenidos de éstas frente a bacterias Gram positivas y Gram negativas de interés clínico, y así contribuir a futuras investigaciones en el área farmacéutica que beneficien la salud humana.

\section{Materiales Y MÉTODOS}

\section{Material biológico}

Se recolectaron 100 muestras planctónicas $(120 \mathrm{~mL})$ en la Bahía Santa Lucía del Puerto de Acapulco (16²9’ N y $99^{\circ} 45^{\prime} \mathrm{O}$ ), desde diciembre 2015 a marzo 2016, utilizando una red de plancton (tipo cono truncado de $1 \mathrm{~m}$ de largo, $50 \mathrm{~cm}$ de diámetro de boca y $54 \mathrm{~mm}$ de abertura de poro) realizando un arrastre superficial circular durante 3 min a una profundidad de $30 \mathrm{~cm}$. La identificación de las especies se realizó con un microscopio óptico binocular (Leica $^{\circledR}, 1349521 X$, Buffalo, N.Y., USA) utilizando los objetivos 10x y 40x y las imágenes obtenidas por microscopía electrónica de barrido (Microscopio Electrónico de Barrido Jeol ${ }^{\circledR}$ T300) con base en las características morfológicas reportadas por Round et al. (2007). Para el aislamiento de las células se empleó el método de pipeta descrito por Andersen (2005).

\section{Cinética de CRECIMiento y PRODUCCión de biomasa}

Se realizaron cultivos monoespecíficos, manteniendo las siguientes condiciones: medio de cultivo F/2 de Guillard (Sigma-Aldrich $^{\circledR}$, St. Louis, MO, USA) en agua marina estéril a una concentración de $4 \mathrm{~mL} \mathrm{~L}^{-1}$, temperatura constante de $25 \pm 0,5^{\circ} \mathrm{C}$, $\mathrm{pH}$ de 7,9 , salinidad de $30-32$ y un fotoperiodo de $12 \mathrm{~h}$ luz/12 h oscuridad. Para monitorear 
el crecimiento celular se realizaron conteos diarios siguiendo la técnica de conteo en cámara de Neubauer, la densidad celular y los parámetros poblacionales se obtuvieron a partir de las siguientes fórmulas (ec. 1-4) (Karlson et al. 2010):

Densidad celular $\left(\right.$ células $\left.\mathrm{mL}^{-1}\right)=\left(\mathrm{N}^{\circ}\right.$ de células totales $/ \mathrm{N}^{\circ}$ de cuadros contados) $* 100^{(1)}$

Velocidad de crecimiento $(\mathrm{K})=(\ln C f-\ln C i) /(t f-t i)^{(2)}$

Tiempo de duplicación $(\mathrm{TD})=\ln 2 / K^{(3)}$

Producción diaria $(\mathrm{PD})=(C f-C i) /(t f-t i)^{(4)}$

Dónde:

$\mathrm{Ci}$ : Concentración inicial

$C f$ : Concentración del cultivo al final de la fase exponencial

$t f-t i$ : Duración de la fase exponencial en días

$\ln$ : Logaritmo natural

La biomasa se recuperó por filtración utilizando un embudo Büchner con papel filtro de $1 \mathrm{~mm}$ de poro en una bomba de vacío, se dejó secar a temperatura ambiente durante $24 \mathrm{~h}$ y se pesó con una balanza analítica (Adventurer Pro AV114C OHAUS, Corporation, Pine Brook, New Jersey, USA).

\section{ObTENCIÓN DE EXTRACTOS}

A partir de la biomasa obtenida se realizaron extracciones mediante el método de maceración con disolventes orgánicos de distinta polaridad (hexano, diclorometano y acetona) durante $24 \mathrm{~h}$. Para evaporar los disolventes se utilizó un rotavapor (Digital 410 Puebla, México) a 70 rpm y temperaturas de 68,39 y $56{ }^{\circ} \mathrm{C}$, respectivamente. Este procedimiento se realizó 2 veces para cada uno de los disolventes y se obtuvo el porcentaje de rendimiento con base en el total de biomasa obtenida (mg).

\section{ACtividad antibacteriana}

Se determinó la actividad biológica de los extractos mediante el método bioautográfico, para lo cual se prepararon dos cromatoplacas de sílica gel ( $\mathrm{N}^{\circ} 60$ Merck) de 5 x $3 \mathrm{~cm}$, sobre el punto de aplicación se colocaron los extractos y fueron eluidas en un sistema hexano: acetona (7:3). Una de las cromatoplacas sirvió como control para la localización de los compuestos, siendo analizada bajo luz ultravioleta (302 y $365 \mathrm{~nm}$ ) y revelada con revelador ácido (10\% ácido sulfúrico, 90\% agua). La segunda cromatoplaca se sumergió en una suspensión de
Staphylococcus aureus ATCC 29213 ajustado al estándar 0,5 de Mc Farland (1,5 x $\left.10^{8} \mathrm{UFC} \mathrm{mL}^{-1}\right)$ durante $30 \mathrm{~s}$ y se incubó a $37{ }^{\circ} \mathrm{C}$ durante $24 \mathrm{~h}$ en cámara húmeda. Transcurrido el tiempo de incubación, el cromatograma se roció con una capa ligera y homogénea de una solución acuosa (3 mg $10 \mathrm{~mL}^{-1}$ de agua destilada) de bromuro de 3(4,5-dimetil-2-tiazoil)-2,5 difeniltetrazolium (MTT, Merk $\AA$, Darmstadt, Germany) como colorante vital y se incubó a $37{ }^{\circ} \mathrm{C}$ durante $30 \mathrm{~min}$. La actividad antibacteriana se interpretó como manchas blancas o zonas de inhibición del crecimiento bacteriano en un fondo violeta (Ríos et al. 1988).

La actividad antibacteriana se determinó por el método de microdilución utilizando las siguientes cepas bacterianas: Staphylococcus aureus ATCC 29213, S. aureus ATCC 25923, S. sciuri ATCC 29061, Enterococcus faecalis ATCC 29212, Enterococcus caseofluvialis ATCC 700327, Enterobacter cloacae ATCC 700323, Escherichia coli ATCC 25922, Pseudomonas aeruginosa ATCC 27853, Klebsiella pneumoniae ATCC 700603 y Salmonella dublin NTCC 9676 estandarizadas al 0,5 de Mc Farland $\left(1,5 \times 10^{8} \mathrm{UFC} \mathrm{mL}^{-1}\right)$. Los extractos se diluyeron con dimetilsulfóxido (DMSO, $200 \mathrm{~mL}$ ) y agua $(800 \mathrm{~mL})$ y se evaluaron a concentraciones de $0,5,1,2,4,8,16$ y $32 \mathrm{mg}$ $\mathrm{mL}^{-1}$ en microplacas de 96 pozos de fondo plano en donde se colocaron $100 \mathrm{~mL}$ de caldo Luria Bertani (LB) y diluciones seriadas de los extractos. Se inocularon $2 \mathrm{~mL}$ de cepas bacterianas y se incubaron a $37^{\circ} \mathrm{C}$ por $24 \mathrm{~h}$. Se usó ceftazidima $\left(10 \mu \mathrm{g} \mathrm{mL}^{-1}\right)$ como control positivo. La placa se leyó utilizando el equipo Thermo Scientific Multiskan GO (Thermo Fisher Scientific Corporation, Vantaa, Finland) ajustado a $600 \mathrm{~nm}$ y analizado con el software SkanIt ${ }^{\mathrm{TM}}$ Software 4.1. Se determinó la Concentración Mínima Inhibitoria (CMI), la cual se define como la concentración más baja en la que el producto natural o extracto inhibe el crecimiento bacteriano (Andrews 2001). Se consideraron activos todos los extractos con concentraciones menores a $8 \mathrm{mg} \mathrm{mL}^{-1}$. Los experimentos fueron realizados por duplicado.

El extracto que presentó las CMI más bajas se probó contra aislados clínicos productores de $\beta$ lactamasas de espectro extendido (BLEE) incluyendo E. coli 2578, E. coli 4036, E. coli 4012 y E. coli 2149 y Staphylococcus coagulasa negativos resistentes a meticilina (SCN-RM) como S. epidermidis 1042, S. haemolitycus 562, S. haemolitycus 731, S. haemolitycus 1036, y S. simulans 1050, usando como controles a Klebsiella pneumoniae ATCC 700603 y Staphylococcus aureus ATCC 01198, con la metodología descrita anteriormente. 


\section{Perfil fitoquímico}

El perfil fitoquímico se realizó mediante cromatografía en capa fina (CCF) de acuerdo a las especificaciones del método bioautográfico (Ríos et al. 1988). Las cromatoplacas se analizaron bajo una lámpara de luz ultravioleta (302 y $365 \mathrm{~nm})$ y se revelaron de acuerdo a lo reportado por Wagner \& Bladt (2001) para alcaloides (Reactivo Dragendorff), flavonoides (Vainillina-ácido clorhídrico), triterpenos (Reactivo de Liebermann-Burchard), aceites esenciales (Vainillina- ácido sulfúrico), lignanos (Vainillina-ácido fosfórico), quinonas y cumarinas (Hidróxido de potasio) y saponinas (Prueba de espuma).

\section{Análisis estadístico}

Todos los resultados se analizaron mediante comparaciones de medias con el análisis de varianza (ANOVA) y Tukey, utilizando el programa IBM ${ }^{\circledR} \mathrm{SPSS}^{\circledR}$ STATISTICS 19 con valores $P<0,05$ para determinar diferencias estadísticas significativas.

\section{Resultados}

Las especies de diatomeas marinas utilizadas en este estudio fueron identificadas con base en la estructura y ornamentación de los frústulos propuesta por Round et al. (2007) y las características morfológicas observadas tanto por microscopio óptico como por microscopía electrónica de barrido. C. curvisetus se caracterizó por presentar espinas terminales no bifurcadas dirigidas hacia el exterior de la curvatura $(22,77 \mathrm{~mm})$ característica de esta especie, presentando un solo plástido, aberturas ovales entre las uniones adyacentes y valvas de forma oval (4,41 x 4,41 mm), se encontraron células individuales y cadenas de 2 a 13 células (Fig. 1A, panel I y II). Para la especie $A$. japonica se observaron colonias en espiral semejando la forma de una estrella con diámetro de $85 \mathrm{~mm}$ formadas por 10 células $(8,42 \times 6,55 \mathrm{~mm})$ de base semitriangular con dos plástidos en su interior, cada célula presentó un ápice largo ligeramente grueso (26,2 mm de longitud) (Fig. 1B, panel I y II). B. mobiliensis se identificó
I

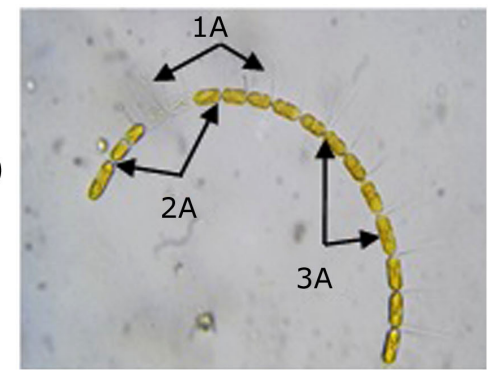

B)

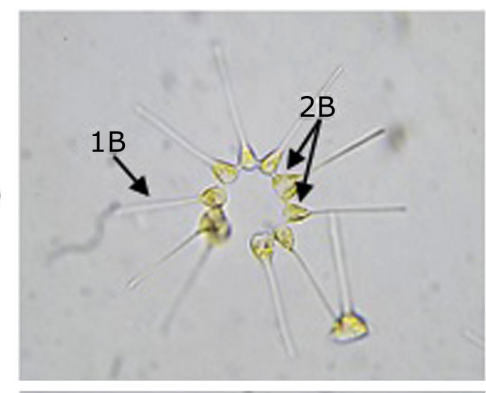

C)

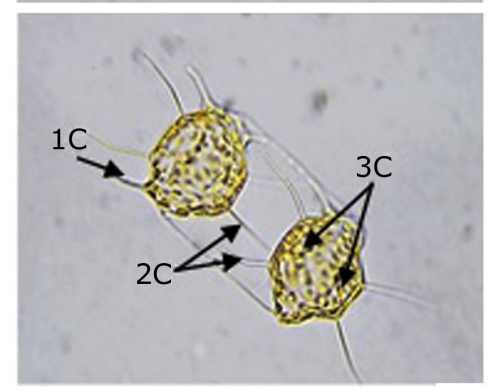

II
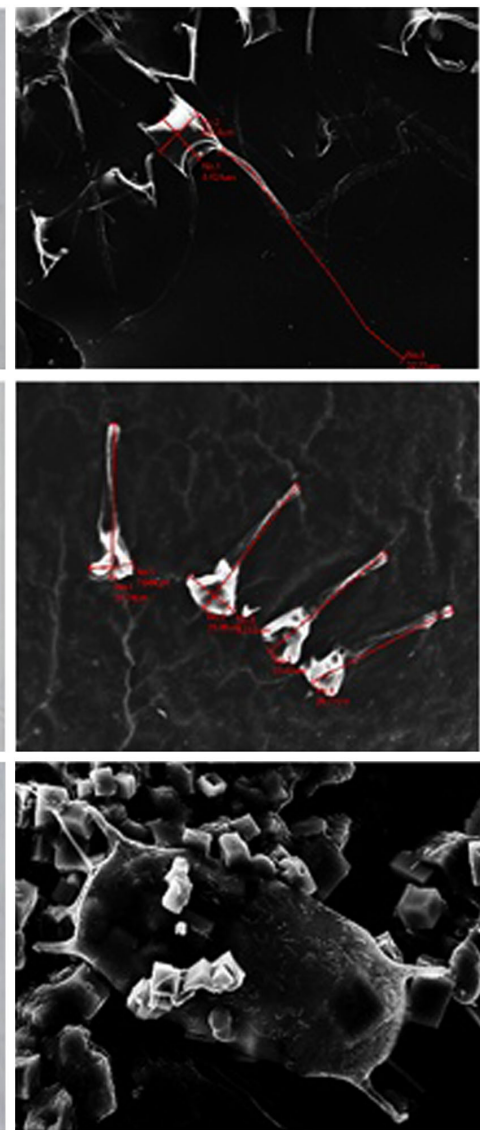

Figura 1. Diatomeas marinas aisladas y cultivadas. A) Chaetoceros curvisetus (1A espinas no bifurcadas, 2A uniones adyacentes, 3A plástidos), B) Asterionella japonica (1B ápice, 2B plástidos) y C) Biddulphia mobiliensis (1C prolongaciones marginales, $2 \mathrm{C}$ espinas centrales, 3 C plástidos). I) Tinción: Lugol. Tomadas en objetivo 40x. II) Imágenes de microscopía electrónica de barrido / Marine diatoms isolated and cultivated. A) Chaetoceros curvisetus (1A nonbifurcated spines, 2A adjacent junctions, 3A plastids), B) Asterionella japonica (1B apex, 2B plastids) and C) Biddulphia mobiliensis ( $1 \mathrm{C}$ marginal extensions, $2 \mathrm{C}$ central spines, 3 C plastids). I) Staining: Lugol. Taken on $40 x$ lens. II) Scanning electron microscopy images 
por sus células oblongas $(170$ x $50 \mathrm{~mm})$ unidas entre sí por espinas centrales largas ligeramente curvadas, presentó prolongaciones marginales cortas, numerosos plástidos, valvas elípticas convexas y numerosas espinas dispersas en el margen (Fig. 1C, panel I y II).

En cuanto a la evaluación de los parámetros cinéticos y poblacionales se demostró que las tres especies poseen una cinética de crecimiento de tipo sigmoidea (Fig. 2). La especie que presentó los mejores parámetros poblacionales estadísticamente significativos $(P<0,05)$ fue $C$. curvisetus con una densidad celular mayor $\left(4,6 \times 10^{4}\right.$ células $\left.\mathrm{mL}^{-1}\right)$ en un menor tiempo de duplicación $[(1,12 \pm 0,02$ días (d)] con una producción diaria de $8.746,67 \pm 70,56$ células $\mathrm{mL}^{-1}$, en comparación con las otras dos especies (Tabla 1) a pesar de tener una velocidad de crecimiento menor $(0,62 \pm 0,01$ d).
La especie con mayor producción de biomasa fue $C$. curvisetus $\left(219,62 \pm 0,99 \mathrm{mg} \mathrm{L}^{-1}\right)$, seguida de $A$. japonica $\left(151,12 \pm 1,41 \mathrm{mg} \mathrm{L}^{-1}\right)$ y B. mobiliensis $\left(109,04 \pm 1,48 \mathrm{mg} \mathrm{L}^{-1}\right)$ en las condiciones probadas. La extracción de biomasa en cada experimento se realizó al inicio de la fase estacionaria, es decir a $\operatorname{los} 4,5$ y 6 días de cultivo respectivamente, donde además se obtuvieron los mayores rendimientos (Tabla 1).

Los porcentajes de rendimiento de los 3 extractos (hexano, diclorometano y acetona) obtenidos de cada una de las especies se muestran en la Tabla 2, en donde se aprecia que el rendimiento de los extractos de diclorometano y acetona de $B$. mobiliensis fueron mayores y estadísticamente significativos $(P<0,05)$, en comparación a los obtenidos para C. curvisetus y $A$. japonica.

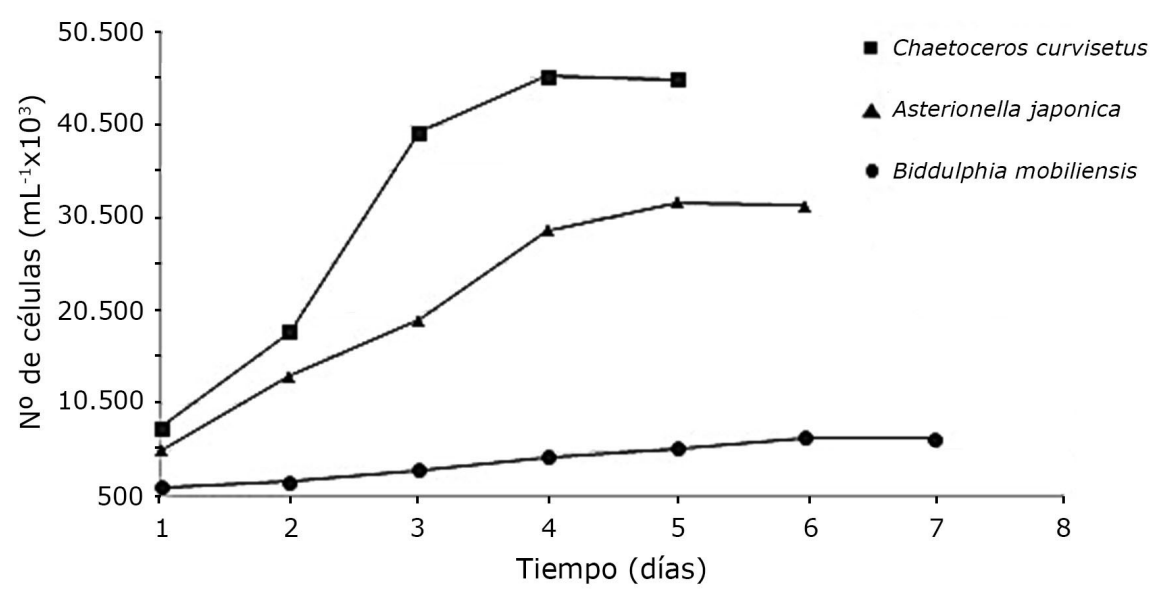

Figura 2. Curvas de crecimiento de tres diatomeas marinas / Growth curves of three marine diatoms

Tabla 1. Parámetros poblacionales de diatomeas marinas y cantidad de biomasa obtenida en base seca \pm DE / Population parameters of marine diatoms and amount of biomass obtained on dry basis \pm SD

\begin{tabular}{|c|c|c|c|c|c|}
\hline \multirow{2}{*}{ Especie } & \multirow{2}{*}{$\begin{array}{l}\text { Densidad celular } \\
\text { (células } \mathrm{mL}^{-1} \text { ) }\end{array}$} & \multicolumn{3}{|c|}{ Parámetros poblacionales } & \multirow{2}{*}{$\begin{array}{l}\text { Biomasa } \\
\left(\mathrm{mg} \mathrm{L}^{-1}\right)\end{array}$} \\
\hline & & $K(d)$ & $\mathrm{TD}(\mathrm{d})$ & PD (células $\mathrm{mL}^{-1}$ ) & \\
\hline Chaetoceros curvisetus & $4,6 \pm 0,54 \times 10^{4 a}$ & $0,62 \pm 0,01^{\mathrm{a}}$ & $1,12 \pm 0,02^{\mathrm{c}}$ & $8746,67 \pm 70,56^{a}$ & $219,62 \pm 0,99^{\mathrm{a}}$ \\
\hline Asterionella japonica & $3,2 \pm 0,55 \times 10^{4 b}$ & $0,47 \pm 0,02^{b}$ & $1,48 \pm 0,04^{b}$ & $5005,56 \pm 44,10^{b}$ & $109,04 \pm 1,48^{\mathrm{c}}$ \\
\hline Biddulphia mobiliensis & $6,9 \pm 0,35 \times 10^{3 \mathrm{c}}$ & $0,40 \pm 0,01^{\mathrm{b}}$ & $1,73 \pm 0,05^{\mathrm{a}}$ & $844,44 \pm 1,84^{\mathrm{c}}$ & $151,12 \pm 1,41^{\mathrm{b}}$ \\
\hline
\end{tabular}

$\mathrm{K}=$ Velocidad de crecimiento, $\mathrm{TD}=$ Tiempo de duplicación y $\mathrm{PD}=$ Producción diaria, $\mathrm{d}=$ días, valores a, $\mathrm{b}$ y c indican diferencias estadísticas (ANOVA y prueba a posteriori de Tukey, $P<0,05: \mathrm{a}>\mathrm{b}>\mathrm{c}$ 
La CMI de la actividad antibacteriana de los extractos se muestra en la Tabla 3, destacando que el extracto con diclorometano de C. curvisetus, presentó actividad contra bacterias ATCC Gram positivas y Gram negativas a concentraciones de 0,5 a $2 \mathrm{mg} \mathrm{mL}^{-1}$. El extracto con acetona de $A$. japonica tuvo mayor actividad antibacteriana, con CMI de $4 \mathrm{mg} \mathrm{mL}^{-1}$ para $S$. aureus ATCC 29213 y $P$. aeruginosa ATCC 27853 y de $8 \mathrm{mg} \mathrm{mL}^{-1}$ para $S$. sciuri ATCC 29061 y E. coli ATCC 25922. Así como el extracto con acetona de $B$. mobiliensis, que mostró una mayor actividad contra bacterias Gram negativas en concentraciones de $0,5 \mathrm{mg} \mathrm{mL}^{-1}$.
Debido a que el extracto de diclorometano de $C$. curvisetus presentó una mayor actividad antibacteriana, se evaluó frente a aislados clínicos productores de BLEE y SCN-RM (Tabla 4) demostrando tener actividad antibacteriana contra K. pneumoniae ATCC 700603 (2 mg $\left.\mathrm{mL}^{-1}\right)$, E. coli $2578\left(8 \mathrm{mg} \mathrm{mL}^{-1}\right)$ y $S$. aureus ATCC 01198 (4 $\mathrm{mg} \mathrm{mL} \mathrm{m}^{-1}$.

Finalmente, en el perfil fitoquímico se demostró que el grupo de compuestos con mayor presencia en todos los extractos evaluados fueron los aceites esenciales, seguidos de alcaloides y compuestos fenólicos (Tabla 5).

Tabla 2. Rendimiento de los extractos totales $(\mathrm{mg})$ de diatomeas marinas / Yield of total extracts $(\mathrm{mg})$ of marine diatoms

\begin{tabular}{lccc}
\hline \multirow{2}{*}{ Diatomea } & \multicolumn{3}{c}{ mg de extracto obtenido \pm DE (\%) } \\
\cline { 2 - 4 } & Hexano & Diclorometano & Acetona \\
\hline Chaetoceros curvisetus & $38,1 \pm 0,69(0,83)^{\mathrm{b}}$ & $205,8 \pm 0,51(4,46)^{\mathrm{b}}$ & $230,9 \pm 0,63(5,01)^{\mathrm{b}}$ \\
Asterionella japonica & $47,5 \pm 1,03(2,07)^{\mathrm{a}}$ & $127,7 \pm 0,68(5,58)^{\mathrm{c}}$ & $195,3 \pm 0,55(8,53)^{\mathrm{c}}$ \\
Biddulphia mobiliensis & $46,3 \pm 0,31(2,02)^{\mathrm{a}}$ & $235,7 \pm 0,68(10,29)^{\mathrm{a}}$ & $286,1 \pm 0,90(9,01)^{\mathrm{a}}$ \\
\hline
\end{tabular}

Valores a, b y c indican diferencias estadísticas (ANOVA y prueba a posteriori de Tukey, $P<0,05: \mathrm{a}>\mathrm{b}>\mathrm{c})$

Tabla 3. Concentración Mínima Inhibitoria (CMI) de los extractos totales de C. curvisetus, A. japonica y B. mobiliensis / Minimum Inhibitory Concentration (MIC) of C. curvisetus, A. japonica and B. mobiliensis extracts

\begin{tabular}{|c|c|c|c|c|c|c|c|c|c|}
\hline \multirow{3}{*}{ Bacterias tipo ATCC } & \multicolumn{9}{|c|}{$\mathrm{CMI}\left(\mathrm{mg} \mathrm{mL}^{-1}\right)$} \\
\hline & \multicolumn{3}{|c|}{$\begin{array}{c}\text { Chaetoceros } \\
\text { curvisetus }\end{array}$} & \multicolumn{3}{|c|}{$\begin{array}{c}\text { Asterionella } \\
\text { japonica }\end{array}$} & \multicolumn{3}{|c|}{$\begin{array}{l}\text { Biddulphia } \\
\text { mobiliensis }\end{array}$} \\
\hline & $\mathrm{H}$ & $\mathrm{D}$ & A & $\mathrm{H}$ & $\mathrm{D}$ & A & $\mathrm{H}$ & $\mathrm{D}$ & A \\
\hline Control negativo * & + & + & + & + & + & + & + & + & + \\
\hline Control negativo** & + & + & + & + & + & + & + & + & + \\
\hline Control positivo*** & 0,5 & 0,5 & 0,5 & 0,5 & 0,5 & 0,5 & 0,5 & 0,5 & 0,5 \\
\hline \multicolumn{10}{|l|}{ Gram positivas } \\
\hline Staphylococcus aureus 29213 & 32 & 0,5 & 8 & 32 & 32 & 4 & 32 & 32 & 8 \\
\hline Staphylococcus aureus 25923 & $>32$ & 1 & $>32$ & 32 & & $>32$ & $>32$ & 32 & $>32$ \\
\hline Staphylococcus sciuri 29061 & $>32$ & 2 & 16 & 32 & $>32$ & 8 & 32 & 32 & 16 \\
\hline Enterococcus faecalis 29212 & 4 & 2 & 16 & $>32$ & 32 & $>32$ & 2 & 2 & $>32$ \\
\hline Enterococcus caseofluvialis 700327 & $>32$ & 2 & $>32$ & 2 & 2 & $>32$ & $>32$ & $>32$ & $>32$ \\
\hline \multicolumn{10}{|l|}{ Gram negativas } \\
\hline Escherichia coli 25922 & $>32$ & 2 & 2 & 32 & 32 & 8 & 32 & 32 & 0,5 \\
\hline Pseudomona aeruginosa 27853 & $>32$ & 0,5 & 16 & 32 & 32 & 4 & 32 & 32 & 0,5 \\
\hline Klebsiella pneumoniae 700603 & $>32$ & 2 & 16 & $>32$ & 2 & 16 & 32 & 32 & 0,5 \\
\hline Enterobacter cloacae 700323 & $>32$ & 2 & $>32$ & $>32$ & 32 & $>32$ & $>32$ & $>32$ & $>32$ \\
\hline Salmonella dublin 9676 & 2 & 2 & 16 & 2 & 32 & $>32$ & 32 & 32 & $>32$ \\
\hline
\end{tabular}

$\mathrm{H}=$ Extracto de hexano, $\mathrm{D}=$ Extracto de diclorometano, $\mathrm{A}=$ Extracto de acetona, $+=$ Crecimiento, $*=$ Bacterias, $* *=$ Bacterias + DMSO, $* * *=$ Ceftazidina $\mu \mathrm{g} \mathrm{mL}^{-1}$ 


\section{DisCusión}

En este estudio se aislaron y cultivaron las especies $C$. curvisetus, A. japonica y B. mobiliensis de la Bahía Santa Lucía del Puerto de Acapulco, Guerrero, México. Las características morfológicas han sido reportados previamente para C. curvisetus (Ishii et al. 2011, Meave del Castillo et al. 2012), para A. japonica (Sunesen et al. 2008), y para B. mobiliensis como lo describen Ojeda (2011) y Lavigne et al. (2015). Las dos primeras especies han sido aisladas con frecuencia en la bahía Santa Lucía de Acapulco, sin embargo, hasta el momento no se han encontrado registros sobre el aislamiento de $B$. mobiliensis en esta bahía, siendo esta la primera vez que se reporta su presencia, lo cual indica que es una especie presente en esta zona costera tropical y su distribución puede estar relacionada con las condiciones ambientales y tróficas, variables a lo largo del año, limitación de nutrientes y patrones de circulación (Alves-de Souza et al. 2008, Meave del Castillo et al. 2012).

C. curvisetus mostró los mejores parámetros tanto cinéticos como poblacionales en comparación a $A$. japonica y $B$. mobiliensis. Esto podría atribuirse a una

Tabla 4. Concentración Mínima Inhibitoria (CMI) del extracto con diclorometano de $C$. curvisetus contra aislados clínicos productores de $\beta$ lactamasas de espectro extendido y Staphylococcus coagulasa negativo resistentes a meticilina / Minimum Inhibitory Concentration (MIC) of the $C$. curvisetus dichloromethane extract in clinical isolates of extended spectrum $\beta$ lactamases and methicillin resistant negative coagulase Staphylococcus

\begin{tabular}{lclc}
\hline Enterobacterias productoras de BLEE & $\begin{array}{c}\mathrm{CMI} \\
\left(\mathrm{mg} \mathrm{mL}^{-1}\right)\end{array}$ & Staphylococcus resistente a meticilina & $\begin{array}{c}\mathrm{CMI}^{-1} \\
\left(\mathrm{mg} \mathrm{mL}^{-1}\right)\end{array}$ \\
\hline Klebsiella pneumoniae ATCC 700603* & 2 & Staphylococcus aureus ATCC 01198** & 4 \\
Escherichia coli 25922*** & 2 & Staphylococcus aureus $29213^{* * * *}$ & 0,5 \\
Escherichia coli 2578 & 8 & Staphylococcus epidermidis 1042 & 32 \\
Escherichia coli 4036 & 16 & Staphylococcus haemolitycus 562 & 32 \\
Escherichia coli 4012 & $>32$ & Staphylococcus haemolitycus 731 & $>32$ \\
Escherichia coli 2149 & $>32$ & Staphylococcus haemolitycus 1036 & $>32$ \\
& & Staphylococcus simulans 1050 & $>32$ \\
\hline
\end{tabular}

BLEE $=\beta$-lactamasa de espectro extendido. ${ }^{*}$ Control positivo de BLEE, $* *$ Control positivo de resistencia a meticilina, $* * *$ Control negativo de BLEE, $* * * *$ Control negativo de resistencia a meticilina

Tabla 5. Perfil fitoquímico cualitativo de $C$. curvisetus, $A$. japonica y $B$. mobiliensis realizado por Cromatografía en Capa Fina (CCF) de los extractos totales / Qualitative phytochemical profile of C. curvisetus, A. japonica and B. mobiliensis by Thin Layer Chromatography (TLC) of the total extracts

\begin{tabular}{lcccccccccc}
\hline \multirow{2}{*}{ Compuestos } & \multicolumn{3}{c}{ C. curvisetus } & \multicolumn{3}{c}{ A. japonica } & \multicolumn{3}{c}{ B. mobiliensis } \\
\cline { 2 - 9 } & $\mathrm{H}$ & $\mathrm{D}$ & $\mathrm{A}$ & $\mathrm{H}$ & $\mathrm{D}$ & $\mathrm{A}$ & $\mathrm{H}$ & $\mathrm{D}$ & $\mathrm{A}$ \\
\hline Saponinas & - & - & + & - & - & + & - & - & + \\
Lignanos & + & - & - & - & - & - & + & - & + \\
Cumarinas & + & + & - & + & + & - & + & - & - \\
Flavonoides & - & - & + & - & - & + & + & + & + \\
Triterpenos & + & + & - & + & + & - & + & + & - \\
Quinonas & + & + & + & - & - & + & + & + & + \\
Alcaloides & - & + & + & - & + & + & + & + & + \\
Aceites esenciales & + & + & + & + & + & + & + & + & + \\
\hline
\end{tabular}

H: extracto de hexano, D: extracto de diclorometano y A: extracto de acetona

$+:$ presencia, -: ausencia 
mejor adaptación fisiológica de esta especie en cultivo in vitro. Sin embargo, los valores en cuanto a densidad celular son menores a lo reportado por Karthikeyan et al.

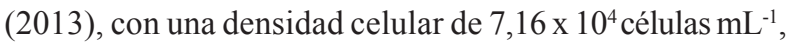
esta diferencia se explica porque se modificaron las concentraciones de los nutrientes con el fin de incrementar intencionalmente la producción celular, mientras que en nuestro estudio no se modificó el medio. Otras especies de Chaetoceros han sido estudiadas, como en el caso de Chaetoceros muelleri, que de acuerdo a lo reportado por Medina-Reyna \& Cordero-Esquivel (1998), presenta una densidad celular de 4,05 x $10^{6}$ células $\mathrm{mL}^{-1}$ a los 5 días de cultivo con una velocidad de crecimiento de 1,31 d, así como Chaetoceros gracilis que presentó una densidad celular de $6,76 \times 10^{4}$ células $\mathrm{mL}^{-1}$ en $8 \mathrm{~d}$ de cultivo con un tiempo de duplicación de 0,038 d y una velocidad de crecimiento de 0,5-3,17 d (Costa et al. 1999), siendo valores mayores a lo reportado en este estudio en comparación a la especie C. curvisetus. De la misma manera, López-Elias et al. (2004) realizaron una comparación de la producción comercial de Chaetoceros sp., en donde se alcanzaron densidades celulares de entre 0,9 y $1,5 \times 10^{6}$ células $\mathrm{mL}^{-1}$ y producciones de biomasa de 9 a $34 \mathrm{~g} \mathrm{~L}^{-1}$ bajo condiciones de cultivo semicontroladas y a diferentes concentraciones de nutrientes.

En la especie $A$. japonica la densidad celular (3,2 x $10^{4}$ células $\left.\mathrm{mL}^{-1}\right)$ es mayor a la reportada por Kain \& Fogg (1958) $\left(2,5 \times 10^{3}\right.$ células $\left.\mathrm{mL}^{-1}\right)$, lo que indica que el medio utilizado y las condiciones ambientales en este trabajo favorece la producción de biomasa y de metabolitos secundarios. Así mismo, Asterionella formosa fue evaluada por Tilman \& Kilham (1976) que modificaron las concentraciones de fosfatos y silicatos, con lo cual obtuvieron un rendimiento de $2,2 \times 10^{6}$ células $\mathrm{mL}^{-1}$ con un tiempo de duplicación de 0,88 d. Por otra parte, Xia et al. (2013) realizaron cultivos de Biddulphia aurita obteniendo una producción de $4 \mathrm{~g} \mathrm{~L}^{-1}$ en 10 días modificando la concentración de nitrógeno y la intensidad de luz. Hasta el momento no se tienen antecedentes específicos que aborden otros parámetros cinéticos como la velocidad de crecimiento, tiempo de duplicación y producción diaria de $B$. mobiliensis, sin embargo la densidad celular $\left(6,9 \times 10^{3}\right.$ células $\left.\mathrm{mL}^{-1}\right)$ fue menor en comparación a la de $C$. curvisetus y $A$. japonica de este estudio. Se requieren más estudios sobre los parámetros población de $B$. mobiliensis ya que produce metabolitos secundarios con alta actividad antibacteriana.
Todas estas variaciones entre las diferentes especies se deben al estrés al que son sometidas las células al ser transferidas a un medio distinto al natural, así como las diferentes condiciones de crecimiento como temperatura, $\mathrm{pH}$, intensidad de luz y las diferentes concentraciones de nutrientes (Hernández-Pérez \& Labbé 2014). Además, se ha demostrado que el crecimiento de las microalgas y/o diatomeas se ve afectado por la fuente de nitrógeno, dado que modulan el transporte y asimilación de nutrientes necesarios para los procesos metabólicos (Matos-Moura et al. 2007). Iriarte et al. (2007) afirman que las formas de nitrógeno reducido (amonio y urea) promueven una mayor biosíntesis de los nutrientes sobre las formas más oxidadas. Se ha demostrado que la manipulación de las condiciones de crecimiento como la concentración de nutrientes, tiempos de incubación, $\mathrm{pH}$, temperatura y otras, tienen un impacto directo en la cantidad y diversidad de la producción de metabolitos secundarios (Bode et al. 2002), por lo que en este estudio la extracción de biomasa se llevó a cabo al inicio de la fase estacionaria ya que de acuerdo a Roze et al. (2011), los metabolitos secundarios son producidos mayoritariamente durante la transición de la fase exponencial a la fase estacionaria. Sin embargo, es necesario investigar la alteración sistemática de los parámetros de cultivo de estas microalgas, con el fin de aumentar el número de metabolitos secundarios con baja polaridad y con alta actividad antimicrobiana.

En cuanto al porcentaje de rendimiento de los extractos totales, se observaron diferencias que se deben al tipo de disolvente utilizado, ya que éstos extraen compuestos de distintos pesos moleculares dependiendo de su estructura química, por ejemplo, los compuestos polifenólicos tienen mayor solubilidad en disolventes polares como la acetona, pero además estos compuestos se dividen en varias clases de acuerdo con el número de anillos aromáticos que contienen, tales como los flavonoides, ácidos fenólicos, estilbenos y lignanos que a su vez tienen pesos moleculares distintos, lo cual se ve reflejado en el peso final del extracto (Ncube et al. 2008).

Se han reportado estudios que evalúan la actividad antibacteriana de C. curvisetus, A. japonica y $B$. mobiliensis, como los reportados por Wang et al. (2010), que evidenciaron la actividad antibacteriana de un extracto obtenido con éter de petróleo frente a Staphylococcus aureus (25 $\left.\mathrm{mg} \mathrm{mL}^{-1}\right)$, Bacillus subtilis, E. 
coli y Proteus vulgaris $\left(12 \mathrm{mg} \mathrm{mL}^{-1}\right)$ siendo concentraciones más elevadas a nuestro estudio. Otro estudio fue el de Sánchez-Saavedra et al. (2010), de un extracto metanólico de C. muelleri, con actividad contra B. subtilis y S. aureus. Además, Sushanth \& Rajashekhar (2015) determinaron la actividad antibacteriana de extractos etanólico, metanólico y hexánico de $C$. calcitrans contra E. coli, S. aureus, Streptococcus pyogenes y B. subtilis. Por otra parte, Viso et al. (1987) demostraron la actividad de A. japonica contra Micrococcus luteus, Sarcina spp. y S. aureus. Así mismo Gauthier (1969), demostró actividad antimicrobiana de Asterionella notata, contra levaduras y hongos filamentosos (Candida albicans, Penicillium sp. y Aspergillus genus). Walter \& Mahesh (2000), determinaron la actividad antibacteriana de un extracto con acetona de $B$. mobiliensis contra $K$. pneumoniae, E. coli, $P$. aeruginosa, Salmonella paratyphi y S. typhy. Sin embargo, todos los autores antes mencionados realizaron la evaluación de la actividad antibacteriana mediante el método de difusión en disco y por lo tanto los resultados no son totalmente comparables.

El hecho de que el extracto con diclorometano de $C$. curvisetus haya mostrado actividad antibacteriana frente a aislados clínicos, es de gran importancia ya que las infecciones causadas por bacterias productoras de BLEE y Staphylococcus RM son un problema mundial que incrementa la morbilidad y mortalidad de los pacientes hospitalizados y en pacientes en la comunidad. E. coli predominantemente como un agente patógeno de infecciones del tracto urinario (Navarro-Navarro et al. 2011, Morones-Esquivel et al. 2016), S. epidermidis en infecciones nosocomiales (Castro-Alarcón et al. 2011, Ehlers et al. 2018) y Klebsiella pneumoniae productoras de carbapenemasas es un importante patógeno relacionado a brotes en centros médicos (RamosCastañeda et al. 2018), por lo que se hace necesario identificar nuevos compuestos que permitan disminuir esta problemática.

El extracto de diclorometano mostró buena actividad contra bacterias ATCC; sin embargo ésta se vio disminuida frente a aislados clínicos (Tablas 3 y 4), esta diferencia puede deberse principalmente a los mecanismos de resistencia presentes en los aislados clínicos, ya que las BLEEs son enzimas que hidrolizan el anillo betalactámico de antibióticos como penicilinas y cefalosporinas de primera, segunda y tercera generación
(Paterson \& Bonomo 2005) y las bacterias RM han generado una variación genética, codificada en el gen mecA, por medio del cual modifican la estructura de su proteína ligadora a penicilina (PBP2a) lo que impide que la antibióticos betalactámicos puedan adherirse al lugar donde va a ejercer la acción bactericida (Louis \& Rice 2006). Una alternativa para incrementar la actividad antibacteriana en aislados clínicos sería evaluar la sinergia del extracto de diclorometano de C. curvisetus y antibióticos comerciales con la finalidad de promover su absorción y potenciar la actividad de estos últimos.

El espectro de actividad de los extractos evaluados se debe al tipo de metabolitos secundarios y su concentración presentes en cada uno de ellos. En el perfil fitoquímico la presencia de aceites esenciales fue visualizada en todos los extractos (Tabla 4), que de acuerdo a Desbois et al. (2009), presentan actividad antibacteriana contra bacterias Gram positivas y Gram negativas, por la capacidad de romper la membrana celular bacteriana. Los aceites esenciales básicamente están compuestos por terpenoides, siendo los sesquiterpenoides y meroterpenoides los que se obtienen con mayor frecuencia de diatomeas marinas (Jyotirmayee et al. 2014). El mecanismo de acción de los terpenoides está dado por el aumento en la permeabilidad de la membrana de las células bacterianas (Griffin 2000). Los ácidos grasos (monoinsaturados y poliinsaturados) también se incluyen entre los aceites esenciales y son producidos por diferentes diatomeas (Lebeau \& Robert 2003), los poliinsaturados se han obtenido con mayor frecuencia de Chaetoceros (Pacheco-Vega et al. 2010, Fuad et al. 2015). Por este motivo, es probable que algunos tipos de ácidos grasos poliinsaturados estén presentes en el extracto total de C. curvisetus y sean estos los responsables de la actividad antibacteriana.

Así mismo, otro grupo de compuestos de gran importancia identificados en este estudio son las saponinas, las cuales son glúcidos que tienen la capacidad de formar complejos con el colesterol presente en las membranas celulares y producir grandes poros en las mismas, alterando la permeabilidad y morfología de la célula microbiana. En las bacterias Gram negativas cuyas paredes celulares están cubiertas por lipopolisacáridos en un $90 \%$, las saponinas tienen la capacidad de interactuar con el lípido A y así aumentar la permeabilidad de la pared celular bacteriana (Arabski et al. 2009), por lo que se sugiere que la actividad antibacteriana del extracto de 
acetona de $B$. mobilienses que presentó los valores de CMI menores contra bacterias ATCC Gram negativas, probablemente esté dada por algún tipo de saponinas.

Los compuestos fenólicos como las quinonas, cumarinas y flavonoides presentan actividad antibacteriana como resultado de la unión a adhesinas, inhibición enzimática, incremento de la permeabilidad, rompimiento de membrana celular, eliminación del sustrato, disipación del potencial de membrana, entre otros mecanismos (Amaro et al. 2011). Así como los terpenos cuya actividad antimicrobiana se ha asociado con la disrupción de la membrana celular bacteriana (Saleem et al. 2010) y los alcaloides, que son compuestos que inhiben el crecimiento de microrganismos patógenos debido a su capacidad de intercalarse con el DNA, de detener la síntesis de proteínas, inducir la apoptosis e inhibir las enzimas del metabolismo de carbohidratos (Sepúlveda-Jiménez et al. 2003).

Sin embargo, la actividad antibacteriana observada en los extractos de diclorometano de $C$. curvisetus, así como los extractos de acetona de A. japonica y B. mobiliensis puede deberse a un efecto sinérgico entre los distintos compuestos y no a uno en particular y además estar relacionada a la presencia de más de un compuesto activo y la concentración de éstos en cada extracto (Coronado et al. 2015). Es importante mencionar que de acuerdo a Rios et al. (1988), se consideran de interés clínico aquellas sustancias puras activas a $100 \mu \mathrm{m} \mathrm{mL}^{-1}$ (menor a las utilizadas en este estudio). Sin embargo, en este estudio se utilizaron extractos totales, con diversos compuestos, por lo cual es necesario analizar la actividad antibacteriana de sus fracciones, que sean utilizadas en menor concentraciones y que pueden ser de interés clínico en futuras investigaciones.

En conclusión, las especies $C$. curvisetus, A. japonica y $B$. mobiliensis pueden cultivarse en medio F/2 Guillard bajo condiciones in vitro favoreciendo la producción de biomasa, siendo este el primer reporte de los parámetros cinéticos de estas diatomeas marinas sin modificaciones en el medio de cultivo. Todos los extractos evaluados presentaron actividad antibacteriana, siendo el extracto con diclorometano de $C$. curvisetus el que más amplio espectro de actividad presentó a las más bajas concentraciones probadas frente a bacterias ATCC Gram positivas y Gram negativas, así como aislamientos clínicos productores de BLEEs. Además, el extracto de acetona de $B$. mobiliensis mostró la mejor actividad frente a bacterias Gram negativas. Por lo anterior se demuestra que estas diatomeas marinas representan una importante fuente de compuestos naturales a los cuales puede atribuirse su actividad biológica antibacteriana y que pueden ser utilizados tanto en la industria farmacéutica como en otros tipos de industria biotecnológica.

\section{Agradecimientos}

El presente estudio se financió a través del fondo para el Fortalecimiento del Posgrado en Biociencias, clave 249671 de los Fondos Mixtos CONACyT Gobierno del Estado de Guerrero. Primer autor contó con beca CONACyT CVU 666408. Se agradece a M.C. Jazmín López Díaz por la toma de las micrografías en el Laboratorio de Microscopía Electrónica de Barrido y Microanálisis (LMEByM) y al C. Víctor Alfonso Cervantes Urieta, auxiliar del Laboratorio de Biotecnología e Impacto Ambiental.

\section{LITERATURA CITADA}

Alves-de-Souza C, MT González \& JL Iriarte. 2008. Functional groups in marine phytoplankton assemblages dominated by diatoms in fjords of southern Chile. Journal of Plankton Research 30:1233-1243.

Amaro H, A Guedes \& F Malcata. 2011. Antimicrobial activities of microalgae: An invited review. Science against microbial pathogen: Communicating current research and technological advances. FORMATEX Microbiology 3(1): 1272-1280.

Andersen RA. 2005. Algal culturing techniques, pp 90-92. Elsevier Academic Press / Phycological Society of America, New York.

Andrews JM. 2001. Determination of minimum inhibitory concentration. Journal of Antimicrobial of Chemotherapy 48(1): 5-16.

Arabski M, S Wasik, K Dworecki \& W Kaca. 2009. Laser interferometric and cultivation methods for measurement of colistin/ ampicilin and saponin interactions with smooth and rough of Proteus mirabilis lipopolysaccharides and cells. Journal of Microbiological Methods 77(2): 178-183.

Bode HB, B Bethe, R Höfs \& A Zeeck. 2002. Big effects from small changes: possible ways to explore nature's chemical diversity. ChemBioChem 2/3(7): 619-627.

Castro-Alarcón N, RM Ribas-Aparicio, J Silva-Sánchez, A Calderón-Navarro, A Sánchez-Pérez, I Parra-Rojas \& G Aparicio-Ozores. 2011. Molecular typing and characterization of macrolide, lincosamide and streptogramin resistance in Staphylococcus epidermidis strains isolated in a Mexican hospital. Journal of Medical Microbiology 60: 730-736. 
Coronado W, L Valerio-González \& H D'Armas. 2015. Tamizaje fitoquímico y actividad antibacteriana preliminar de los extractos de la macroalga Laurencia dendroidea, J. Agardh, 1841 (Rhodomelaceae: Rhodophyta). SABER. Revista Multidisciplinaria del Consejo de Investigación de la Universidad de Oriente 27(1): 61-66.

Costa AA, ML Koening \& JM Sílvio. 1999. Use of secondary sewage water as a culture medium for Chaetoceros gracilis and Thalassiosira sp. (Chrysophyceae) in laboratory conditions. Brazilian Archives of Biology and Technology 42(2): 739-540. < doi 10.1590/S1516-89131999000200011>

Desbois A, A Mearns-Spragg \& V Smith. 2009. A fatty acid from the diatom Phaeodactylum tricornutum is antibacterial against diverse bacteria including multi-resistant Staphylococcus aureus (MRSA). Marine Biotechnology 11(1):45-52.

Ehlers MM, W Strasheim, M Lowe, V Ueckermann \& MM Kock. 2018. Molecular epidemiology of Staphylococcus epidermidis implicated in catheter-related bloodstream infections at an Academic Hospital in Pretoria, South Africa. Frontiers in Microbiology 9: 417. <doi: 10.3389/ fmicb.2018.00417>

Fuad MAM, N Mohammad-Noor, AKC Jalal \& BY Kamaruzzaman. 2015. Growth profile and fatty acid accumulation of four Chaetoceros taxa isolated from coastal water of Pahang, Malaysia. Sains Malaysiana 44(8): 10771084.

Gauthier M. 1969. Antibacterial activity of a marine diatom: Asterionella notata. Revue Internationale d'Océanographie et Medicale 15(16):103-171.

Gómez-Luna L. 2007. Microalgas: aspectos ecológicos y biotecnológicos. Revista Cubana de Química 19: 3-20.

Griffin S. 2000. Aspects of antimicrobial activity of terpenoids and the relationship to their molecular structure. Thesis Doctor of Philosophy, University of Western Sidney, Penrith, 301 pp.

Hernández-Becerril DU. 2014. Biodiversidad de algas planctónicas marinas (Cyanobacteria, Prasinophyceae, Euglenophyta, Chrysophyceae, Dictyochophyceae, Eustigmatophyceae, Parmophyceae, Raphidophyceae, Bacillariophyta, Cryptophyta, Haptophyta, Dinoflagellata) en México. Revista Mexicana de Biodiversidad 85: 44-53.

Hernández-Pérez A \& JI Labbé. 2014. Microalgae, culture and benefits. Revista de Biología Marina y Oceanografía 49(2): 157-173.

Iriarte JL, RA Quiñones, RR González \& CP Valenzuela. 2007. Relación entre actividad enzimática y biomasa de ensambles fitoplanctónicos en el sistema pelágico. Investigaciones Marinas 35(1): 71-84.
Ishii K-I, M Iwataki, K Matsuoka \& I Imai. 2011. Proposal of identification criteria for resting spores of Chaetoceros species (Bacillariophyceae) from a temperate coastal sea. Phycologia 50: 351-362.

Jyotirmayee P, D Sachidananda \& KD Basanta. 2014. Antibacterial activity of freshwater microalgae: A review. African Journal of Pharmacy and Pharmacology 8(32): 809818.

Kain JM \& GE Fogg. 1958. Studies on the growth of marine phytoplankton I Asterionella japonica Gran. Journal of the Marine Biological Association of United Kingdom 37: 397-413.

Karlson B, C Cusack \& E Bresnan. 2010. Microscopic and molecular methods for quantitative phytoplankton analysis. Intergovernmental Oceanographic Commission of UNESCO, IOC Manuals and Guides 55: 29-30.

Karthikeyan P, K Manimaran, P Sampathkumar \& L Rameshkumar. 2013. Growth and nutrient removal properties of the diatoms, Chaetoceros curvisetus and $C$. simplex under different nitrogen sources. Applied Water Science (3): 49-55.

Lavigne A, I Sunesen \& E Sar. 2015. Morphological, taxonomic and nomenclatural analysis of species of Odontella, Trieres and Zygoceros (Triceratiaceae, Bacillariophyta) from Anegada Bay (Province of Buenos Aires, Argentina). Diatom Research 30(4): 307-331.

Lebeau T \& JM Robert. 2003. Diatom cultivation and biotechnologically relevant products. Part II: Current and putative products. Applied Microbiology and Biotechnology 60: 624-632.

Lee SD \& JH Lee. 2011. Morphology and taxonomy of the planktonic diatom Chaetoceros species (Bacillariophyceae) with special intercalary setae in Korean coastal waters. Algae 26(2): 153-165.

López-Elías JA, D Voltolina, M Nieves-Soto \& L FigueroaOrtiz. 2004. Producción y composición de microalgas en laboratorios comerciales del Noroeste de México. En: Cruz Suárez LE, D Ricque-Marie, MG Nieto-López, D Villarreal, U Scholz \& M González (eds). Avances en nutrición acuícola VII. Memorias del VII Simposio Internacional de Nutrición Acuícola, 16-19 Noviembre, Hermosillo, Sonora, México, pp. 636-649.

Louis B \& MD Rice. 2006. Antimicrobial resistance in GramPositive bacteria. The American Journal of Medicine 119(6A): S11-S19.

Matos-Moura A, E Bezerra-Neto, ML Koening \& E Eskinazi-Leca. 2007. Chemical composition of three microalgae species for possible use in mariculture. Brazilian Archives of Biology and Technology 50(3): 461-467. 
Meave del Castillo ME, ME Zamudio-Resendiz \& M Castillo-Rivera. 2012. Riqueza fitoplanctónica de la Bahía de Acapulco y zona costera aledaña, Guerrero, México. Acta Botánica Mexicana 100: 405-487.

Medina-Reyna CE \& B Cordero-Esquivel. 1998. Crecimiento y composición bioquímica de la diatomea Chaetoceros muelleri Lemmerman, mantenida en cultivo estático en un medio comercial. Ciencia y Mar 2(6): 19-25.

Moreno-Díaz G, AA Rojas-Herrera, J González-González, J Violante-González, JL Rosas-Acevedo \& S GarcíaIbáñez. 2015. Variación temporal de la abundancia y composición de especies de fitoplancton colectado con red, en la Bahía de Acapulco, México. Revista Bio Ciencias 3(2): 88-102.

Morones-Esquivel I, TG Salgado-Muñoz, TI GonzagaLópez, AP Matamoros-Mejía, JO Terán-González, S Arteaga-Vázquez, LJ Castro-D'Franchis, AE ReyesJiménez, DS López-González \& D Meza-Oviedo. 2016. Enterobacterias con betalactamasas de espectro extendido en hemocultivos y urocultivos. Medicina Interna de México 32(4): 381-387.

Navarro-Navarro M, RE Robles-Zepeda, A Garibay-Escobar \& E Ruiz-Bustos. 2011. Escherichia coli y Klebsiella pneumoniae comunitarias y hospitalarias productoras de $\beta$-lactamasas en hospitales de Hermosillo, Sonora. Salud Pública de México 53: 341-344.

Ncube NS, AJ Afolayan \& AI Okoh. 2008. Assessment techniques of antimicrobial properties of natural compounds of plant origin: current methods and future trends. African Journal of Biotechnology 7(12): 1797-1806.

Ojeda A. 2011. Diatomeas de las aguas costeras de las Islas Canarias. Instituto Canario de Ciencias Marinas, Agencia Canaria de Investigación, Innovación y Sociedad de la Información Gobierno de Canarias. <http://mdc.ulpgc.es/ $\mathrm{cdm} / \mathrm{ref} /$ collection/MDC/id/156053>

Pacheco-Vega JM, MA Cadena-Roa, MP SánchezSaavedra, D Tovar-Ramírez \& CR Dávalos. 2010. Effect of culture medium and nutrient concentration on fatty acid content of Chaetoceros muelleri. Revista Latinoamericana de Biotecnología Ambiental y Algal 1: 6-15.

Pal R \& AK Choudhury. 2014. An introduction of phytoplanktons: Diversity and ecology, $167 \mathrm{pp}$. Springer India, New Delhi.

Paterson DL \& RD Bonomo. 2005. Extended spectrum $\beta$ lactamases: a clinical updated. Clinical Microbiology Reviews 18: 657-686.

Ramos-Castañeda JA, A Ruano-Ravina, R Barbosa-Lorenzo, JE Paillier-Gonzalez, JC Saldaña-Campos, DF Salinas \& EV Lemos-Luengas. 2018. Mortality due to KPC carbapenemase-producing Klebsiella pneumoniae infections: Systematic review and meta-analysis: Mortality due to KPC Klebsiella pneumoniae infections. Journal of Infection 76(5): 438-448. <doi: 10.1016/j.jinf.2018.02.007>
Ríos JL, MC Recio \& A Villar. 1988. Screening methods for natural products with antimicrobial activity: a review of the literature. Journal of Ethnopharmacology 23: 127-149.

Rojas-Herrera AA, J Violante-González, VMG SevillaTorres, JS Gil-Guerrero, P Flores-Rodríguez \& JA Rendón-Dircio. 2012. Species composition and abundance of phytoplankton communities in Acapulco Bay, Mexico. International Research Journal of Microbiology 3(9): 307316.

Round FE, RM Crawford \& DG Mann. 2007. Diatoms: Biology and morphology of the genera, pp. 350-351. Cambridge University Press, Cambridge.

Roze LV, A Chanda \& JE Linz. 2011. Compartmentalization and molecular traffic in secondary metabolism: a new understanding of established cellular processes. Fungal Genetic and Biology 48: 35-48.

Sánchez-Saavedra M, A Licea-Navarro \& J BernáldezSarabia. 2010. Evaluation of the antibacterial activity of different species of phytoplankton. Revista de Biología Marina y Oceanografía 45(3): 531-536.

Saleem M, M Nazir, MS Ali, H Hussain, YS Lee, N Raiz \& A Jabbar. 2010. Antimicrobial natural products: an update on future antibiotic drug candidates. Natural Product Reports 27: 238-254.

Sepúlveda-Jiménez G, H Porta-Ducoing \& M Rocha-Sosa. 2003. La participación de los metabolitos secundarios en la defensa de las plantas. Revista Mexicana de Fitopatología 21(3): 355-363.

Sunesen I, DU Hernández-Becerril \& EA Sar. 2008. Marine diatoms from Buenos Aires coastal waters (Argentina). V. species of the genus Chaetoceros. Revista de Biologia Marina y Oceanografia 43(2): 303-326.

Sushanth VR \& M Rajashekhar. 2015. Antioxidant and antimicrobial activities in the four species of marine microalgae isolated from Arabian Sea of Karnataka coast. Indian Journal of Geo-Marine Sciences 44(1): 69-75.

Tilman D \& SS Kilham. 1976. Phosphate and silicate growth and uptake kinetics of the diatoms Asterionella formosa and Cyclotella meneghiniana in batch and semicontinuos culture. Journal of Phycology 12: 375-383.

Viso AC, D Pesando \& C Baby. 1987. Antibacterial and antifungal properties of some marine diatoms in culture. Botanica Marina 30: 41-45.

Volkman JK, G Eglinton \& EDS Corner. 1980. Sterols and fatty acids of the marine dinoflagellate Biddulphia sinensis. Phytochemistry 19: 1809-1813.

Wagner H \& S Bladt. 2001. Plant drug analysis: a thin layer chromatography atlas, 384 pp. Springer Verlag, Berlin / Heidelberg / New York.

Walter CS \& R Mahesh. 2000. Antibacterial and antifungal activities of some marine diatoms in culture. Indian Journal of Marine Sciences 29: 238-242. 
Wang Q, C Liu, S Zhang, L Sun \& C Wang. 2010. Extraction and antimicrobial effect of active components from Chaetoceros curvisetus. Food Science 31(5): 180-183.
Yongmanitchai W \& OP Ward. 1989. Omega-3 fatty acids: alternative sources of production. Process Biochemistry 24: 117-125.

Xia S, K Wang, L Wan, A Li, Q Hu \& C Zhang. 2013.

Production, Production, characterization and antioxidant activity of fucoxanthin from the marine diatom Odontella aurita. Marine Drugs 11(7): 2667-2681.

Recibido el 12 de mayo de 2017 y aceptado el 19 de julio de 2018

Editor: Claudia Bustos D. 